\title{
Real-world experience with pembrolizumab in patients with advanced soft tissue sarcoma
}

\author{
Jiayong Liu", Zhengfu Fan", Chujie Bai, Shu Li, Ruifeng Xue, Tian Gao, Lu Zhang, Zhichao Tan, \\ Zhiwei Fang
}

Department of Bone and Soft Tissue Tumor, Peking University Cancer Hospital, Key laboratory of Carcinogenesis and Translational Research (Ministry of Education/Beijing), Beijing, China

Contributions: (I) Conception and design: J Liu, Z Fan; (II) Administrative support: Z Fan; (III) Provision of study materials or patients: J Liu, C Bai, Z Fang; (IV) Collection and assembly of data: S Li, T Gao; (V) Data analysis and interpretation: L Zhang, Z Tan; (VI) Manuscript writing: All authors; (VII) Final approval of manuscript: All authors.

\#These authors contributed equally to this work.

Correspondence to: Jiayong Liu, MD. Department of Bone and Soft Tissue Tumor, Peking University Cancer Hospital, Key laboratory of Carcinogenesis and Translational Research (Ministry of Education/Beijing), 52, Fucheng Rd., Beijing 100142, China. Email: liujiayong_doc@163.com.

Background: The goal of this study was to retrospectively analyze the efficacy and safety of pembrolizumab in the real-world treatment of soft tissue sarcoma (STS).

Methods: We analyzed 38 patients who suffered from STS and received pembrolizumab treatment from July 2017 to December 2018 in our hospital. We investigated the influence of clinical characteristics, treatment timing, and treatment protocol on objective response rate (ORR). We also investigated the factors affecting overall survival (OS) and progression-free survival (PFS), as well as the occurrence of severe adverse events (SAEs).

Results: The overall ORR was 19.4\% (7/36). The ORRs of patients who received pembrolizumab treatment as first-line, second-line, and third-line therapy were $42.9 \%(3 / 7), 25.0 \%(4 / 16)$, and $0 \%$ $(0 / 13)$, respectively, which showed marginal significance $(\mathrm{P}=0.052)$. Four patients $(11.1 \%)$ maintained a complete response (CR) or partial response (PR) for at least 6 months with pembrolizumab monotherapy, or after withdrawal of chemotherapy or targeted therapy regimens. The median PFS was 2.9 months [95\% confidence interval (CI): 2.4-3.4 months] and the median OS was 12.0 months (95\% CI: 10.2-13.8 months). Cox regression analysis showed that treatment time was an independent factor affecting $\mathrm{PFS}(\mathrm{P}=0.041)$, while Eastern Cooperative Oncology Group (ECOG) performance status (PS) score was the only independent factor affecting OS $(\mathrm{P}=0.028)$.

Conclusions: In the real world, the effectiveness of pembrolizumab in the treatment of STS was low. Some subtypes showed a limited response to pembrolizumab, including alveolar soft part sarcoma (ASPS), undifferentiated pleomorphic sarcoma (UPS), exoskeletal chondrosarcoma (ESCS), and angiosarcoma (AS), while the response in leiomyosarcoma (LMS) was low. Combination therapy may increase the risk of SAEs, especially when combined with pazopanib.

Keywords: Soft tissue sarcoma (STS); pembrolizumab; immunotherapy; target therapy; chemotherapy; real world

Submitted Dec 03, 2020. Accepted for publication Feb 05, 2021.

doi: $10.21037 / \mathrm{atm}-21-49$

View this article at: http://dx.doi.org/10.21037/atm-21-49 


\section{Introduction}

At present, there is still a lack of effective protocols for the treatment of advanced soft tissue sarcoma (STS), and patient prognosis is poor. The first-line treatment for most sarcoma subtypes is anthracycline-based chemotherapy, which can achieve an objective responsive rate (ORR) of $20-40 \%$ and disease control in 2.3 to 4.9 months (1-5). After failure of first-line chemotherapy, there is no standard recommended second-line treatment. Some new chemotherapeutic agents, including gemcitabine, trabectedin, and eribulin, have shown some benefit for certain subtypes of STS, but their significance for improving overall survival (OS) is still controversial (6-8). Some tyrosine kinase inhibitors (TKIs), including pazopanib, sunitinib, regorafenib, and anlotinib, amongst others, were reported to offer some benefit for disease control, but there are no large-scale randomized studies which demonstrate their effectiveness in improving OS (9-12). In the PALETTE study, the median progressionfree survival (PFS) was 4.6 months for patients receiving pazopanib compared with 1.6 months for the placebo group $(\mathrm{P}<0.0001)$, but there was no significant difference in OS between the 2 groups (12.5 vs. 10.7 months, $\mathrm{P}=0.25$ ) (9).

Recently, immune checkpoint inhibitors (ICIs), including pembrolizumab, have made great breakthroughs in cancer therapy, enabling some patients with advanced melanoma, lung cancer, and bladder cancer to achieve long-term disease control (13-15). However, except for some specific cancers, the response rate of solid tumors to immunotherapy is generally low. Therefore, how to screen for potential patients and how to improve the efficacy of ICIs through combination therapies are 2 promising research directions in the future. The molecular markers reported to be related to the therapeutic effect of ICIs include the expression of programmed death-ligand 1 (PD-L1) on tumor cells, tumor mutation burden, microsatellite instability, tumor infiltrating lymph node cells, and neoplastic antigen, among others (14-18). In addition, some clinical trials have shown that combining chemotherapy, targeted therapy, and radiotherapy can improve the response rate to ICIs (19-22).

Only a few studies have reported on the efficacy and safety of ICIs for STS $(23,24)$. The results of the SARC028 trial showed that the ORR of pembrolizumab for the treatment of STS was $18 \%$. The efficacy of pembrolizumab was reported to be related to the subtype of sarcomas, as it had a relatively high ORR in undifferentiated pleomorphic sarcoma (UPS) (23) and alveolar soft part sarcoma (ASPS) $(24,25)$. Unfortunately, there is no evidence that most molecular markers, including PD-L1, are associated with the therapeutic effect of ICIs. The low incidence of STS and the high variance of responses make research into immunotherapies complex and difficult (26). This study retrospectively analyzed patients with advanced STS treated with pembrolizumab at our hospital, and investigated the potential factors related to its efficacy and safety.

We present the following article in accordance with the STROBE reporting checklist (available at http://dx.doi. org/10.21037/atm-21-49).

\section{Methods}

\section{Patient information}

We examined 38 patients with pathologically confirmed STS who received pembrolizumab immunotherapy at Peking University Cancer Hospital \& Institute from July 2017 to December 2018. All procedures performed in this study involving human participants were in accordance with the Declaration of Helsinki (as revised in 2013). Informed consent was taken from all the patients. This study was approved by the Ethics Committee of Peking University Cancer Hospital. (No. 2019YJZ02). All patients had metastatic disease and had measurable lesions according to the Response Evaluation Criteria in Solid Tumors (RECIST) v1.1 standards (18). The Eastern Cooperative Oncology Group (ECOG) performance status (PS) scores were 0-2. Pathological subtypes included leiomyosarcoma (LMS), UPS, ASPS, synovial sarcoma (SS), pleomorphic rhabdomyosarcoma (PRMS), clear cell sarcoma (CCS), dedifferentiated liposarcoma (DDLS), epithelioid sarcoma (ES), malignant peripheral nerve sheath tumor (MPNST), angiosarcoma (AS), carcinosarcoma, exoskeletal chondrosarcoma (ESCS), and desmoplastic small round cell tumor (DSRCT). Based on previous systemic treatments, pembrolizumab was administered in 8 patients as first-line therapy, in 17 patients as second-line therapy, and in 13 patients as third- or further-line therapy. PD-L1 expression was evaluated if the tumor specimen can be obtained, using the proprietary Dako 22C3 IHC assay. PD-L1 expression was determined as positive if it was expressed in more than $1 \%$ of tumor cells.

\section{Groups and therapy}

Patients were divided into a pembrolizumab monotherapy group (A), combined chemotherapy group (B), and 
combined targeted therapy group (C), according to their medication strategy. Group A received pembrolizumab injections at $2 \mathrm{mg} / \mathrm{kg}$ for 21 days per cycle. In group $\mathrm{B}$, the standard dose of anthracycline-based regimens (AIM, anthracycline, ifosfamide and mesna; or AD, anthracycline and dacarbazine), high dose ifosfamide, or gemcitabine combined with docetaxel or dacarbazine were used simultaneously with pembrolizumab injection. Chemotherapy was repeated for 4 to 6 cycles, or was discontinued if the disease progressed or intolerable adverse events (AEs) occurred. In group $\mathrm{C}$, in addition to pembrolizumab, the patients were treated with either anlotinib (12 mg/day for 14 consecutive days, then discontinued for 7 days, in a 21-day cycle), pazopanib (400-600 mg/day), or lenvatinib $\left(10-18 \mathrm{mg} / \mathrm{m}^{2}\right)$ simultaneously. Target agents were consistently used until disease progression, intolerable toxicity, or complete tumor remission occurred. For all patients, pembrolizumab was continued for up to 2 years or until disease progression or unacceptable AEs occurred.

\section{Treatment outcomes and adverse reactions}

Radiographic tumor assessment, including magnetic resonance imaging or computed tomography, was performed at baseline and every 6 to 8 weeks during treatment. Safety assessments, including routine blood and biochemical tests, analysis of thyroid function and myocardial enzymes, and electrocardiograms, were performed at baseline and every 3 weeks before pembrolizumab administration. Special examinations were performed if the patient had suspicious AEs related symptoms. For example, lung CT scans were recommended if dyspnea and cough were present. The adverse reactions of patients were recorded and scored during follow-up. The primary endpoint was ORR, and the secondary endpoints included PFS, OS, and AEs. To further evaluate the effect of pembrolizumab alone, we assessed disease status 6 months after the withdrawal of chemotherapy and targeted therapy in groups B and C. ORR was defined as the ratio of complete response (CR) and partial response (PR) according to RECIST standards (18). PFS was defined as the time from the beginning of treatment to disease progression or death from cancer. OS was defined as the time from the beginning of treatment to patient death. AEs were classified according to the National Cancer Institute Common Terminology Criteria for Adverse Events, version 4.0 (27).

\section{Statistical analysis}

SPSS 22.0 software was used for statistical analysis. Count data was expressed as frequency and percentage. The relationships between ORR and different therapeutic methods, treatment lines, and clinical characteristics were tested using Pearson's chi-square test. Comparisons between PFS and OS were analyzed by Kaplan-Meier survival curves, and the Cox regression model was used to analyze related factors affecting PFS and OS. $\mathrm{P}<0.05$ (twotailed) was defined as statistically significant.

\section{Results}

\section{Basic characteristics of patients}

From July 2017 to December 2018, 38 patients with advanced STS received immunotherapy with pembrolizumab in our hospital. Among them, 17 were males and 21 were females, with an average age of 42.6 years (12-79 years). According to the ECOG PS assessments, 5 cases $(13.2 \%)$ scored 0,20 cases $(52.6 \%)$ scored 1 , and 13 cases $(34.2 \%)$ scored 2 . The number of cases of each pathological subtype were 9 LMS, 5 ASPS, 4 UPS, 4 PRMS, 4 CCS, 4 DDLS, 2 SS, 1 ES, 1 MPNST, 1 AS, 1 carcinosarcoma, 1 ESCS, and 1 DSRCT. Six cases received pembrolizumab monotherapy, including 2 cases receiving it as first-line treatment, 2 cases receiving it sequentially after chemotherapy, 1 case receiving it after TKI-targeted therapy, and 1 case with a history of both chemotherapy and targeted therapy. Another 16 cases received pembrolizumab combined with chemotherapy, including 7 cases with $\mathrm{AD} /$ AIM and 9 cases with other regimens, and 5 cases were treated with pembrolizumab combined with chemotherapy as first-line therapy, 8 cases were treated as second-line therapy, and 3 cases were treated as third- or further-line therapy. Of the 16 patients treated with pembrolizumab combined with targeted therapy, 8 patients received pazopanib, 6 received anlotinib, and 2 received lenvatinib. Only 1 of these patients was treated with pembrolizumab combined with targeted therapy as first-line therapy, while 7 cases were treated as second-line therapy, and 8 cases were treated as third- or further-line therapy.

\section{Therapeutic response}

All patients were treated with pembrolizumab for more 


\section{Page 4 of 12}

Table 1 The relationship between treatment response and clinical characteristics

\begin{tabular}{|c|c|c|c|}
\hline Basic feature & Case number & ORR & $P$ value \\
\hline Sex & & & 0.418 \\
\hline M & 15 & $4(26.7 \%)$ & \\
\hline $\mathrm{F}$ & 21 & $3(14.3 \%)$ & \\
\hline Age & & & 0.209 \\
\hline$\leq 40$ & 14 & $1(7.1 \%)$ & \\
\hline$>40$ & 22 & $6(27.3 \%)$ & \\
\hline ECOG performance & & & 0.642 \\
\hline 0 & 6 & $2(33.3 \%)$ & \\
\hline 1 & 18 & $3(16.7 \%)$ & \\
\hline 2 & 12 & $2(16.7 \%)$ & \\
\hline LDH & & & 0.652 \\
\hline Elevated & 9 & $1(11.1 \%)$ & \\
\hline Normal & 27 & $6(22.2 \%)$ & \\
\hline PD-L1 & & & 0.235 \\
\hline Negative & 9 & $1(11.1 \%)$ & \\
\hline Positive & 6 & $3(50 \%)$ & \\
\hline NA & 21 & $3(14.3 \%)$ & \\
\hline Treatment time & & & 0.052 \\
\hline First-line & 7 & $3(42.9 \%)$ & \\
\hline Second-line & 16 & $4(25.0 \%)$ & \\
\hline Third-line or more & 13 & $0(0 \%)$ & \\
\hline Therapeutic methods & & & 0.996 \\
\hline Monotherapy & 5 & $1(20.0 \%)$ & \\
\hline Chemotherapy combined & 16 & $3(18.8 \%)$ & \\
\hline Target therapy combined & 15 & $3(20.0 \%)$ & \\
\hline
\end{tabular}

ECOG, Eastern Cooperative Oncology Group; ORR, objective responsive rate; $\mathrm{LDH}$, lactate dehydrogenase; PD-L1, programmed cell death-ligand 1; NA, not available.

than 2 cycles. Two patients died within 2 months without evaluation of treatment response. Of the remaining 36 patients, 3 patients survived less than 12 weeks and were evaluated at the 6 th week, while the rest of the patients were evaluated every 6-8 weeks. One patient (2.8\%) achieved CR, 6 patients (16.7\%) achieved PR, 17 patients (47.2\%) experienced stable disease (SD), and 12 patients (33.3\%) experienced progressive disease (PD). The overall

\section{Liu et al. Pembrolizumab for advanced soft tissue sarcoma}

ORR was $19.4 \%(7 / 36)$. The ORRs of patients who received the treatments as first-line, second-line, or thirdline therapy were $42.9 \%(3 / 7), 25.0 \%$ (4/16), and $0 \%$ $(0 / 13)$, respectively, which showed marginal significance $(\mathrm{P}=0.052)$. However, there was no significant difference between different combination treatment groups $(\mathrm{P}=0.996)$, with an ORR of $20.0 \%(1 / 5), 18.8 \%$ (3/16), and $20.0 \%$ $(3 / 15)$ in groups A, B, and C, respectively. Other clinical features, including sex, age, ECOG PS score, and lactate dehydrogenase (LDH) level, were also not associated with ORR (Table 1). PD-L1 was detected in 15 patients, among which 6 patients (40\%) had positive PD-L1 expression. No significant correlation was found between treatment efficacy and PD-L1 expression levels $(\mathrm{P}=0.235$, Table 1$)$.

Four patients (11.1\%) maintained CR or PR for at least 6 months with pembrolizumab monotherapy or after withdrawal of chemotherapy or targeted therapy regimens (Table 2), including 1 case each of ASPS, UPS, ESCS, and AS (Figure 1). However, none of the patients with LMS, the subtype with the highest number of cases (9), achieved long-term disease control. No factors were found to be associated with this indicator.

\section{Survival conditions}

All patients were followed for a median follow-up period of 11.9 months (range, 1.4-31.3 months). The median PFS was 2.9 months [95\% confidence interval (CI): 2.4-3.4 months]. There were significant differences in PFS between patients with different lines of treatment, with a median PFS of 5.8 months (95\% CI: 2.6-11.0 months), 3.5 months (95\% CI: 2.0-5.0 months), and 2.8 months (95\% CI: 2.4-3.1 months) for patients receiving treatment as first-line, second-line, and thirdor further-line therapy, respectively $(\mathrm{P}=0.003$; Figure $2 A)$. The median PFS of patients with normal LDH was 3.2 months $(95 \%$ CI: 2.3-4.2 months), which was significantly higher than the PFS of 2.1 months (95\% CI: 1.3-2.9 months) for patients with elevated LDH $(\mathrm{P}=0.025$; Figure $2 B)$. No significant difference in PFS was detected between treatment groups $\mathrm{A}, \mathrm{B}$, and $\mathrm{C}$ ( $\mathrm{P}=0.160,2.8$ vs. 4.2 vs. 2.8 months, respectively; Figure 2C). Other clinical characteristics, including age, sex, ECOG PS score, and PD-L1 expression, did not significantly affect PFS.

The median OS was 12.0 months (95\% CI: 10.2-13.8 months). There were significant differences in OS between patients with different lines of treatment, with a median OS of 19.1 months (95\% CI: 11.9-26.3 months), 15.4 months (95\% CI: 12.8-19.8 months), and 7.0 months (95\% CI: 4.19.9 months) for patients receiving treatment as first-line, 
Table 2 Detailed information of the 4 patients who maintained PR or SD for at least 6 months with pembrolizumab

\begin{tabular}{cccccccccc}
\hline Sex & Age & Subtype & ECOG score & LDH & Treatment time & PD-L1 & Treatment group & Therapeutic response Time to progression \\
\hline M & 52 & ASPS & 2 & 0 & Second-line & NA & $\begin{array}{c}\text { Pembrolizumab } \\
\text { alone }\end{array}$ & PR & Not reached \\
F & 45 & UPS & 0 & 0 & Second-line & NA & Combined AD & PR & Not reached \\
M & 79 & ESCS & 2 & 1 & Second-line & Positive & $\begin{array}{c}\text { Combined } \\
\text { lenvatinib }\end{array}$ & PR & CR \\
M & 47 & AS & 1 & 0 & First-line & Positive & Combined AD & Not reached \\
\hline
\end{tabular}

ECOG, Eastern Cooperative Oncology Group; PR, partial response; CR, complete response; SD, stable disease; PD-L1, programmed cell death-ligand 1; LDH, lactate dehydrogenase; ASPS, alveolar soft part sarcoma; UPS, undifferentiated pleomorphic sarcoma; ESCS, exoskeletal chondrosarcoma; AS, angiosarcoma; NA, not available; AD, anthracycline and dacarbazine.
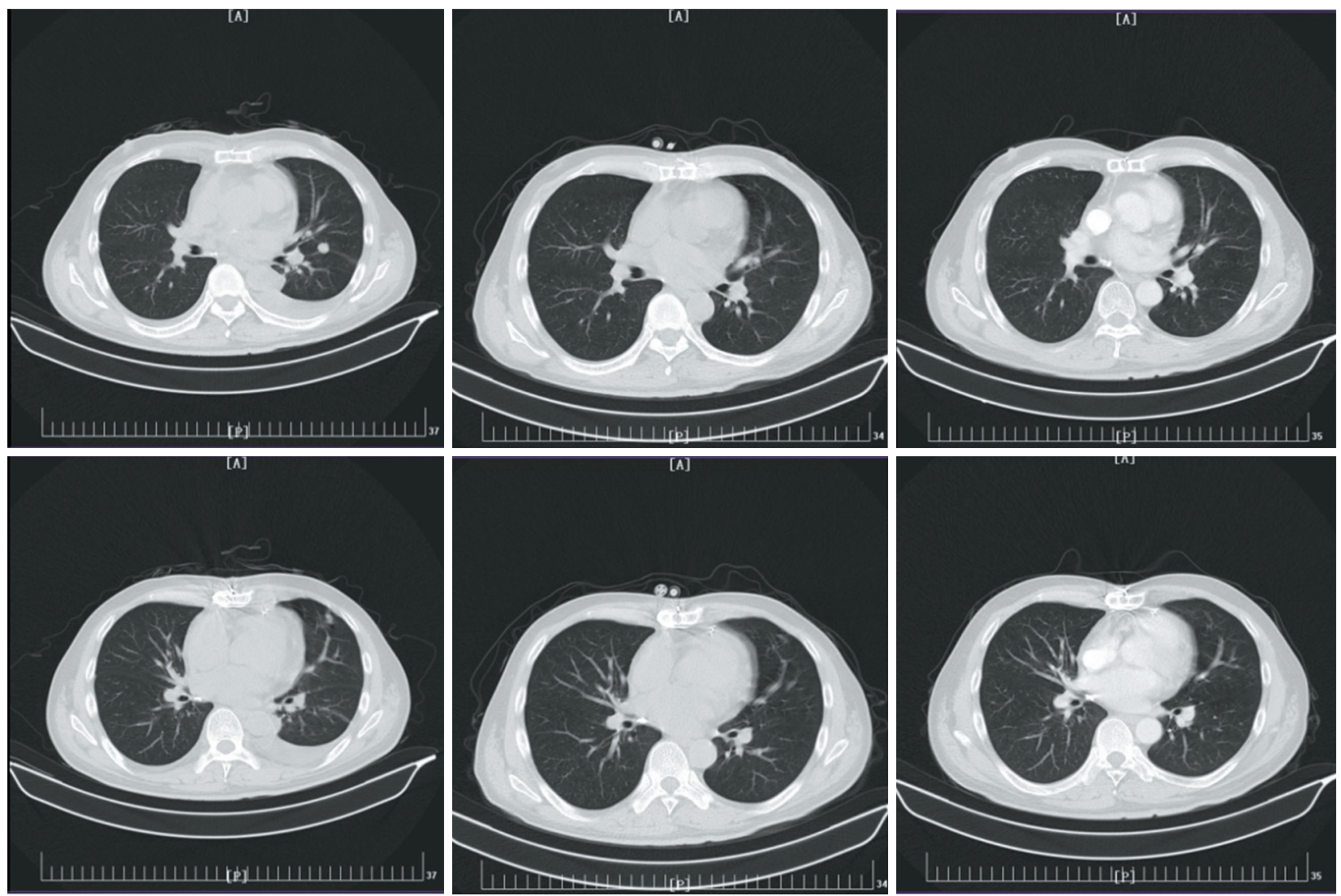

Figure 1 A patient with cardiac angiosarcoma achieved complete response (CR) after treatment with pembrolizumab combined with $\mathrm{AD}$ (anthracycline and dacarbazine) chemotherapy. (A) In a patient with cardiac angiosarcoma, pulmonary metastasis and hydrothorax were found after resection of the primary lesion. (B) When the patient started combined pembrolizumab and AD chemotherapy, the lung lesions were significantly reduced and the hydrothorax disappeared 6 cycles later on December 14, 2018. (C) The patient maintained clinical CR after maintenance with pembrolizumab monotherapy for 11.5 months through to November 29, 2019. 


\section{Page 6 of 12}

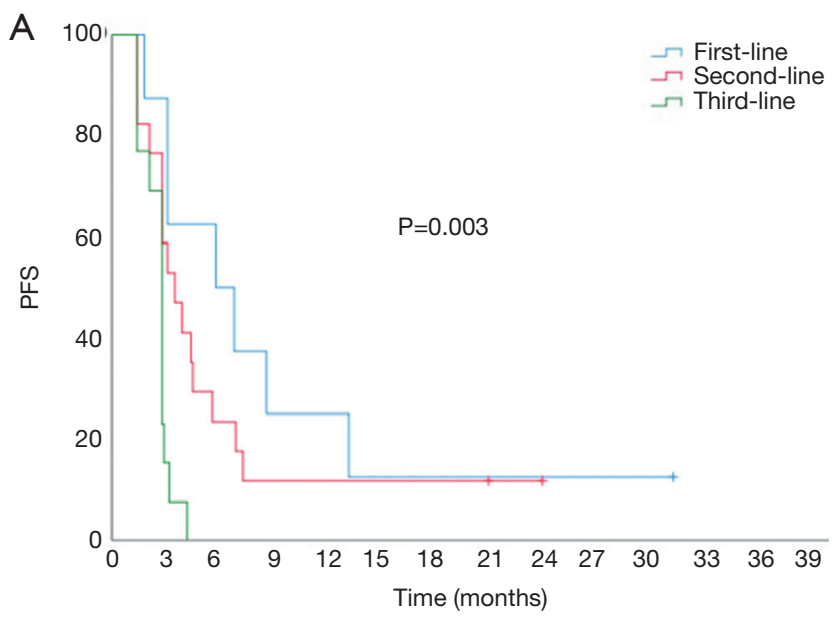

B

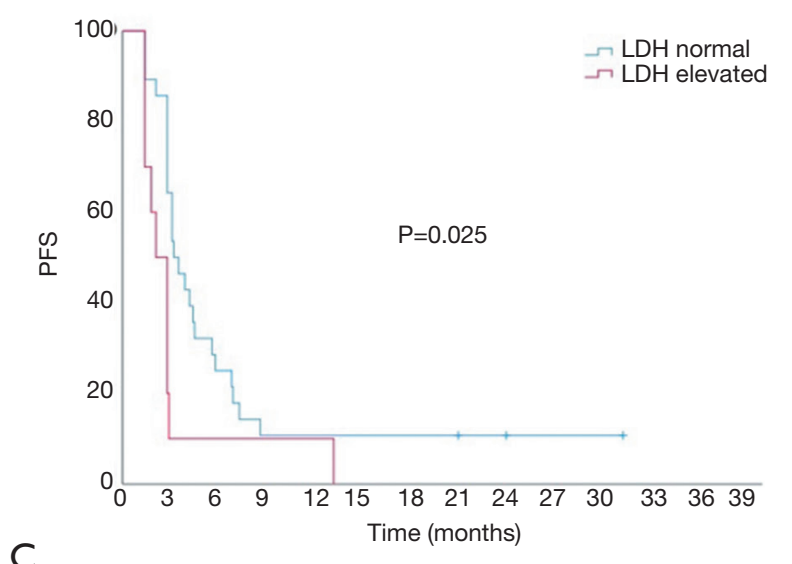

C

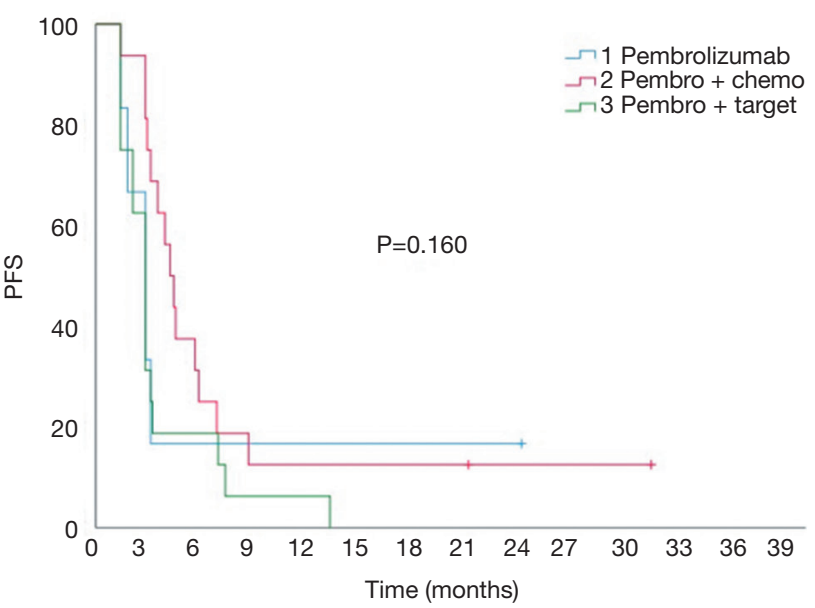

Figure 2 Kaplan-Meier univariate analysis of progressionfree survival (PFS). (A) Influence of treatment time on PFS. (B) Influence of serum lactate dehydrogenase (LDH) level on PFS. (C) Influence of combination strategies on PFS.

\section{Liu et al. Pembrolizumab for advanced soft tissue sarcoma}

second-line, and third- or further-line therapy, respectively $(\mathrm{P}=0.002$; Figure $3 A)$. ECOG PS also significantly affected OS, with a median OS of 25.2 months (95\% CI: 19.930.5 months), 12.5 months (95\% CI: 7.7-17.3 months), and 7.5 months (95\% CI: 4.8-10.2 months) in patients with a score of 0,1 , and 2 , respectively $(\mathrm{P}=0.005$; Figure $3 B)$. The median OS of patients with normal serum LDH was significantly longer than those with elevated LDH (14.4 vs. 7.5 months, $\mathrm{P}=0.007)$. There were no significant differences in OS between the different treatment groups $(\mathrm{P}=0.185$, Figure 3C). A Cox regression model of PFS was established by incorporating treatment line, therapeutic strategy, and LDH level (Table 3). The results showed that treatment time was the only independent factor affecting PFS. The risk of disease progression after treatment with ICIs as thirdor further-line therapy was 3.94 times higher than that for first-line treatment (95\% CI: $1.05-11.66, \mathrm{P}=0.041)$. A Cox regression model of OS was established by incorporating treatment time, ECOG PS score, and LDH level (Table 3). The results showed that the ECOG PS score was the only independent factor affecting OS. The risk of death in patients with an ECOG PS score of 2 was 10.6 times greater than for those with a score of 0 . The risk of death in patients treated with third- or further-line therapy was 2.75 times the risk for patients treated with first-line therapy, with a marginal statistical difference (95\% CI: 0.88-8.59; $\mathrm{P}=0.057$ ).

\section{Adverse events}

The most common adverse reaction was fever, with an incidence of $68.4 \%(26 / 38)$, of which 6 cases $(16.3 \%)$ had a temperature exceeding $38.5^{\circ} \mathrm{C}$. The other common adverse reactions were leukopenia $(34.2 \%, 13 / 38)$, diarrhea $(26.3 \%, 10 / 38)$, hypothyroidism $(24.3 \%, 9 / 37)$, abnormal liver function $(18.4 \%, 7 / 38)$, oral ulceration (18.4\%, 7/38), and creatine kinase elevation (13.2\%, $5 / 38$ ). Six patients (15.8\%) had severe AEs (SAEs), which led to treatment discontinuation (Table 4). In 1 patient with ASPS, platelets decreased to less than $1 \times 10^{9} / \mathrm{L}$ after 2 cycles of pazopanib combined with pembrolizumab. The patient died of gastrointestinal bleeding despite a large dose of glucocorticoids and platelet transfusion. Because it has not been reported that pazopanib can cause severe thrombocytopenia, which occurred in this patient very suddenly and rapidly, it was thought that 


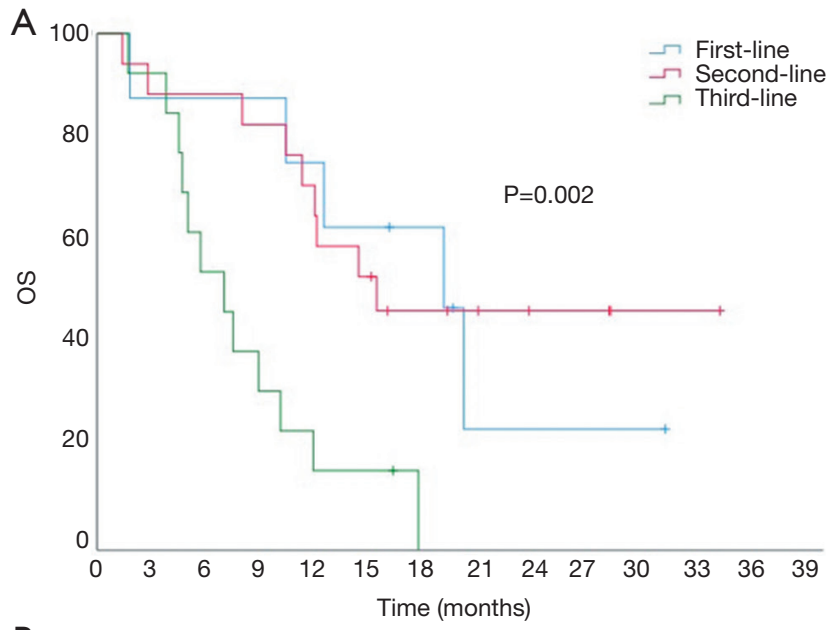

B

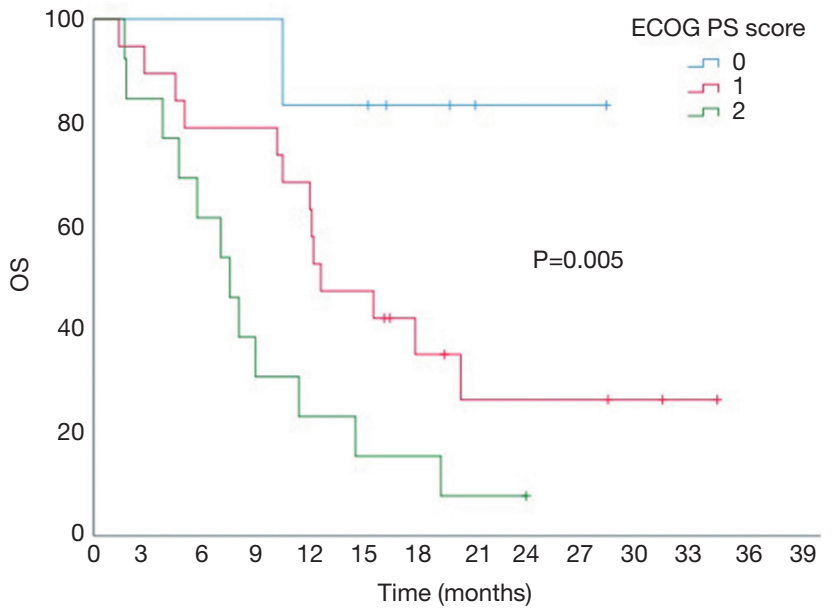

C

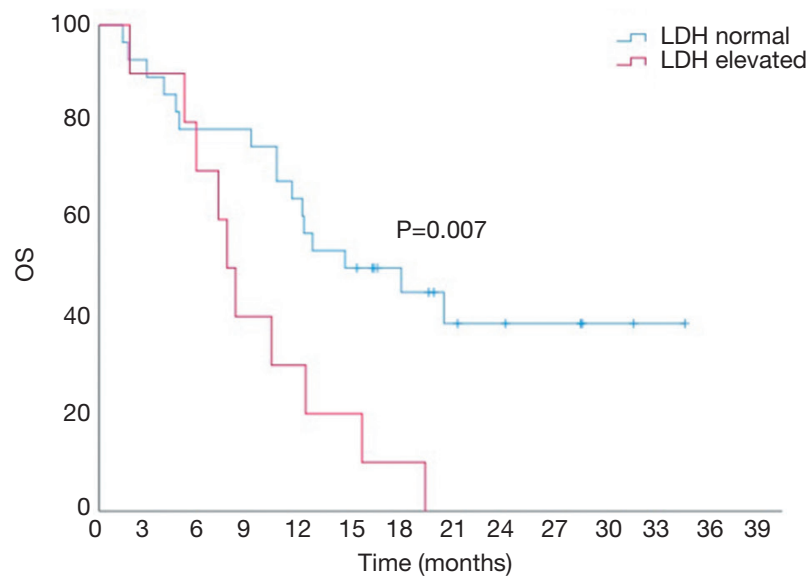

Figure 3 Kaplan-Meier univariate analysis of overall survival (OS). (A) Influence of treatment time on OS. (B) Influence of combination strategies on OS. (C) Influence of serum lactate dehydrogenase (LDH) level on OS. the thrombocytopenia was related to the combination of pazopanib with pembrolizumab. In another case, a patient with PRMS developed very severe systemic skin bullae after using nonsteroidal anti-inflammatory drugs (NSAIDs) during the 4th cycle of pembrolizumab combined with AIM chemotherapy. We finally decided after discussion with dermatologists that the reaction was epidermolysis bullosa induced by the ICI, and after administration of a glucocorticoid and topical potassium permanganate, the skin bullae completely healed and only mild pigmentation remained. A patient with ASPS developed emergent blindness after 2 cycles of pembrolizumab combined with pazopanib. After discontinuation of both drugs and administration of glucocorticoids, symptoms improved completely within 2 days. Although no fundus examination was performed, the blindness was determined to be related to pembrolizumab. One patient had grade 4 bilirubin elevation, which returned to grade 2 after discontinuation of pembrolizumab and cortisol treatment. Unfortunately, the patient died of heart failure 2 months later due to old age and poor cardiopulmonary function. The other 2 cases of SAEs were pneumonia and liver function injury. After withdrawal of pembrolizumab and treatment with methylprednisolone, symptoms were relieved in both patients. All 6 SAEs occurred in patients receiving combination therapy, including 2 cases $(10.5 \%)$ receiving pembrolizumab combined with chemotherapy and 4 cases (23.5\%) receiving pembrolizumab combined with targeted therapy.

\section{Discussion}

ICI immunotherapy has made breakthroughs in the treatment of advanced solid tumors. However, the response rate of single-drug immunotherapy is still low in individual tumors. The SARC028 study showed that the response rate to pembrolizumab in the treatment of STS was $18 \%$, and the response of osteosarcoma was only $5 \%(2 / 40)(23)$. The overall response rate of the patients in the present study was $19.4 \%$, but this was achieved by combination with chemotherapy or immunotherapy in most patients. The proportion of patients who remained stable or showed PR for more than 6 months after discontinuing combination agents was only $11.1 \%$, which may be a better indicator of the real efficacy of pembrolizumab. This ratio was lower than in the SARC028 trial, and the PFS and OS of 
Table 3 Cox regression model and multivariate analysis results

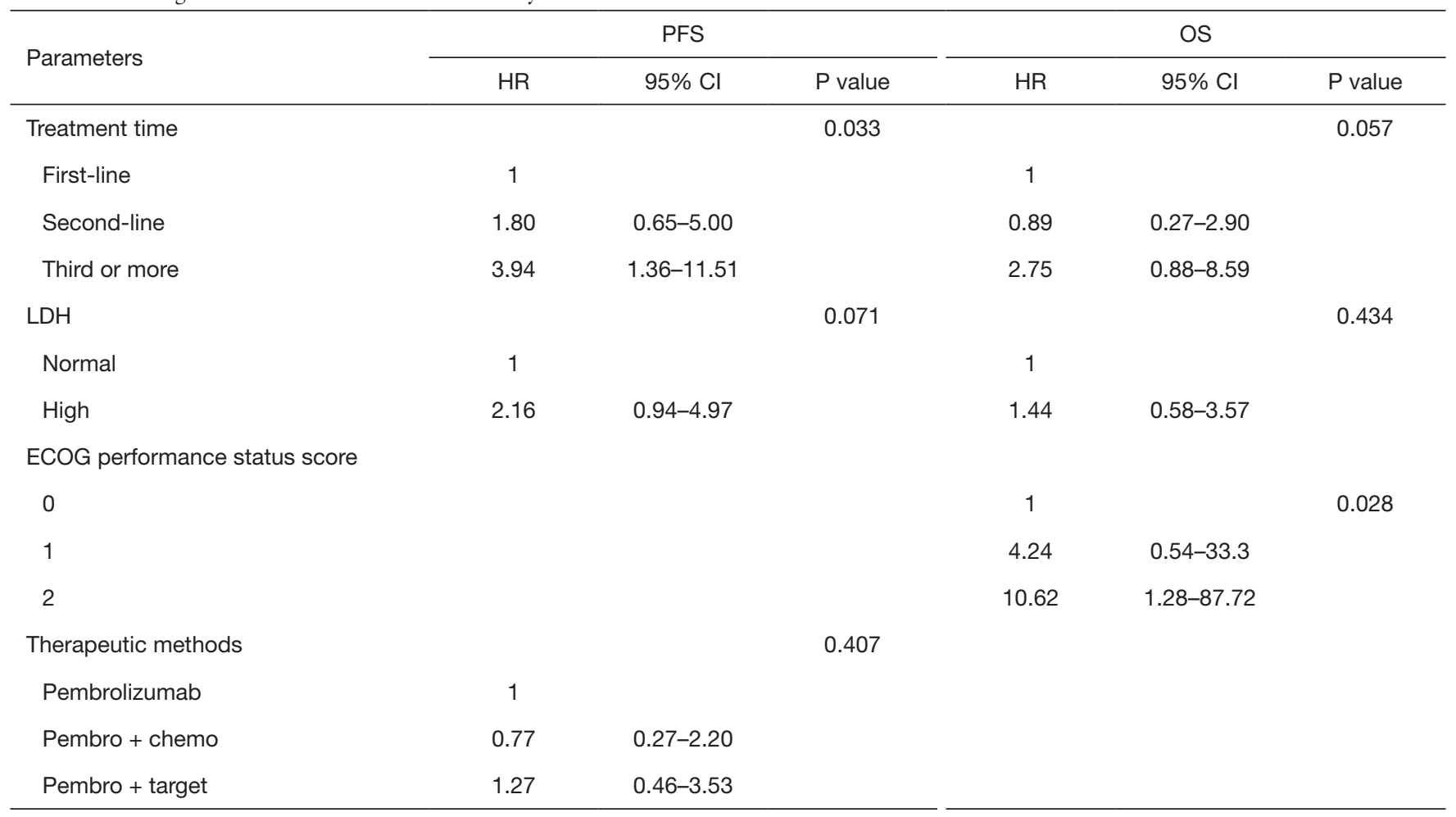

ECOG, Eastern Cooperative Oncology Group; PFS, progression-free survival; OS, overall survival; Cl, confidence interval; HR, hazard ratio; $\mathrm{LDH}$, lactate dehydrogenase.

patients in our cohort were also relatively shorter. The most likely reason behind this finding may be that many patients had poor general health status and had received multiple lines of systemic therapy before administration of immunotherapy. It has been reported that the ECOG score can affect the effectiveness of pembrolizumab, and both ORR and survival in patients with high ECOG scores were lower than in those with low ECOG scores $(28,29)$. In addition, the time of treatment also had a significant impact on the effectiveness of pembrolizumab. In nonsmall cell lung cancer (NSCLC) patients with a PD-L1 tumor proportion score $\geq 50 \%$, the median OS of those who received pembrolizumab as first-line therapy in the KEYNOTE-024 study was 30.0 months, while the OS of patients treated with pembrolizumab as second-line therapy in KEYNOTE-010 was only 10.4 months $(28,30)$. In the real world, most sarcoma patients do not use ICIs early when they develop metastasis because there are no indications. However, due to the lack of effective therapeutic methods, many patients have tried pembrolizumab at a later stage. In the present study, $33.3 \%$ of patients had an ECOG PS score of 2, 50.0\% had an ECOG PS score of 1, and only $19.4 \%$ of patients received pembrolizumab as first-line therapy. Although the sample size was small, the chi-square test showed that time of treatment was a probable related factor affecting ORR, and the survival analysis indicated that it was also an independent factor affecting PFS and OS, while ECOG PS score mainly affected OS.

In this study, no factors were found to be associated with the effectiveness of pembrolizumab, including the expression of PD-L1 and different therapeutic combinations. In fact, no clear molecular marker associated with an immunotherapeutic effect on STS has been identified so far. It was reported that although PD-L1 was positive in $30-50 \%$ of STS, it was not found to be associated with the therapeutic effect of pembrolizumab $(31,32)$. In a pooled analysis of a phase II trial with 384 sarcoma patients, the ORR and non-progression rates were similar in patients treated with anti-PD-1/PD-L1 as a single agent and those treated with a combination regimen (33). Different subtypes have different response rates to ICIs. Published studies have confirmed that ASPS and UPS have a high response 
Table 4 Occurrence of severe adverse reactions

\begin{tabular}{|c|c|c|c|c|c|c|c|}
\hline Sex & Age & Subtype & Treatment time & Treatment group & $\begin{array}{c}\text { Therapeutic } \\
\text { response }\end{array}$ & Side effects & Outcome of treatment \\
\hline M & 54 & PRMS & First-Line & $\begin{array}{l}\text { Combined with } \\
\text { chemotherapy (AIM) }\end{array}$ & PR & Epidermolysis bullosa, G4 & $\begin{array}{l}\text { Recovered after cortisol } \\
\text { treatment }\end{array}$ \\
\hline M & 79 & Carcinosarcoma & First-line & $\begin{array}{l}\text { Combined with } \\
\text { anlotinib }\end{array}$ & NA & Elevated bilirubin, G4 & $\begin{array}{l}\text { Recovered and died of heart } \\
\text { failure after } 1.8 \text { months }\end{array}$ \\
\hline $\mathrm{F}$ & 41 & CCS & Second-line & $\begin{array}{l}\text { Combined with } \\
\text { pazopanib }\end{array}$ & PD & $\begin{array}{l}\text { Transaminase elevated, G3; } \\
\text { elevated bilirubin, G2 }\end{array}$ & $\begin{array}{l}\text { Recovered after } \\
\text { cortisol treatment }\end{array}$ \\
\hline $\mathrm{F}$ & 53 & LMS & Second-line & $\begin{array}{l}\text { Combined with } \\
\text { chemotherapy (AIM) }\end{array}$ & SD & Pneumonia & $\begin{array}{l}\text { Recovered after } \\
\text { cortisol treatment }\end{array}$ \\
\hline
\end{tabular}

PRMS, pleomorphic rhabdomyosarcoma; ASPS, alveolar soft part sarcoma; CCS, clear cell sarcoma; LMS, leiomyosarcoma; NA, not available; AIM, doxorubicin + ifosfamide + mesna; AD, doxorubicin + dacarbazine; G, grade; SD, stable disease; PD, progressive disease; $\mathrm{PR}$, partial response

rate to pembrolizumab $(23,25)$, but only a few cases of other subtypes have been reported. In this study, we found 2 cases of AS and ESCS in which the efficacy lasted more than 6 months. Because the incidence of these 2 subtypes is very low, whether they have a high response rate to pembrolizumab needs to be confirmed in a multi-center clinical study with a large sample size. In addition, among the 9 cases of LMS in this study, there was no case with efficacy lasting more than half a year, suggesting that LMS may have a low response rate to ICIs. Given the inherent heterogeneity of sarcoma, future studies should examine histological subtypes to better understand the mechanisms of resistance and response.

The most common adverse reaction we observed in this study was fever $(68.4 \%)$. The rates of other adverse reactions were all below $35 \%$. However, 6 patients (15.8\%) had SAEs which led to treatment discontinuation, but the organs involved showed no specific pattern, and included liver, lung, bone marrow, skin, and eyes. The incidence of SAEs in this study was higher than that reported in other studies, ranging from $5-8 \%(23,34,35)$. In a metaanalysis of immune-related AEs (irAEs), which included 12,808 oncologic patients treated with anti-PD-1/PDL1 agents, the overall incidence of irAEs was $26.82 \%$ in any grade and $6.10 \%$ for severe grade irAEs (34). The main reason for the increase in SAEs may be related to the combination strategies. All 6 cases of SAEs in this cohort occurred in the combination treatment groups, including 2 cases with combined chemotherapy and 4 cases with combined targeted agents, accounting for $10.5 \%$ and $23.5 \%$ of cases in each group, respectively. In patients with NSCLC, discontinuation of treatment due to AEs was more frequent in the pembrolizumab-combination group than in the placebo-combination group (13.3\% vs. 6.4\%) (36). Combinations with TKIs can also increase the incidence of SAEs, but the incidence of SAEs with different TKIs combined with pembrolizumab varies greatly. In a clinical trial of renal cell cancer, the percentage of AEs leading to treatment discontinuation reached as high as $80 \%$, and the trial was subsequently terminated (37). However, pembrolizumab combined with axitinib or lenvatinib showed favorable safety, with only $9-21 \%$ of patients discontinuing study treatment because of treatmentrelated AEs $(24,38)$. In the present study, there were 3 cases of SAEs which occurred after combined treatment with pazopanib, confirming the high risk of this combination. Because pazopanib is currently the only FDA-approved broad-spectrum TKI used for non-adipogenic STS, its safety should be strongly considered when combining with ICIs. The efficacy and safety of other TKIs combined with ICIs in the treatment of STS need to be confirmed in further clinical trials.

Our study was limited by its retrospective design and small sample size. In addition, 13 sarcoma subtypes were 
included in this study, which made it more difficult to analyze the efficacy and factors related to pembrolizumab. Therefore, we were careful when interpreting our findings. For example, in order to exclude the influence of other therapeutic factors on pembrolizumab, we assessed the disease status 6 months after withdrawal of chemotherapy and targeted agents in patients who received combination therapy. However, we did produce some interesting findings. For example, in addition to ASPS and UPS, pembrolizumab was effective in AS and ESCS, but not in LMS, which had the largest number of cases. Furthermore, we found that the major treatment-related SAEs occurred in patients receiving combination therapy, especially those receiving combinations with pazopanib. The low incidence of STS and the high variance of responses are the biggest obstacles to clinical research in this field. Although the results of this study were mostly descriptive, these data may be useful in optimizing future clinical trials of STS and guiding clinical practice.

\section{Conclusions}

In the real world, the effectiveness of pembrolizumab in the treatment of STS has been shown to be low. Some subtypes had a better response rate to pembrolizumab, including ASPS, UPS, ESCS, and AS, while the response of LMS was low. The PFS and OS of the patients in this group were poor, which was related to late treatment times and poor ECOG PS scores. Combination therapy with pembrolizumab may increase the risk of SAEs, especially when combined with pazopanib.

\section{Acknowledgments}

Funding: China International Medical Foundation (No. Z-2014-06-15331).

\section{Footnote}

Reporting Checklist: The authors have completed the STROBE reporting checklist. Available at http://dx.doi. org/10.21037/atm-21-49

Data Sharing Statement: Available at http://dx.doi. org/10.21037/atm-21-49

Conflicts of Interest: All authors have completed the ICMJE uniform disclosure form (available at http://dx.doi. org/10.21037/atm-21-49). The authors have no conflicts of interest to declare.

Ethical Statement: The authors are accountable for all aspects of the work in ensuring that questions related to the accuracy or integrity of any part of the work are appropriately investigated and resolved. All procedures performed in this study involving human participants were in accordance with the Declaration of Helsinki (as revised in 2013). Informed consent was taken from all the patients. This study was approved by the Ethics Committee of Peking University Cancer Hospital. (No. 2019YJZ02).

Open Access Statement: This is an Open Access article distributed in accordance with the Creative Commons Attribution-NonCommercial-NoDerivs 4.0 International License (CC BY-NC-ND 4.0), which permits the noncommercial replication and distribution of the article with the strict proviso that no changes or edits are made and the original work is properly cited (including links to both the formal publication through the relevant DOI and the license). See: https://creativecommons.org/licenses/by-nc-nd/4.0/.

\section{References}

1. Lorigan P, Verweij J, Papai Z, et al. Phase III trial of two investigational schedules of ifosfamide compared with standard-dose doxorubicin in advanced or metastatic soft tissue sarcoma: a European Organisation for Research and Treatment of Cancer Soft Tissue and Bone Sarcoma Group Study. J Clin Oncol 2007;25:3144-50.

2. Palumbo R, Neumaier C, Cosso M, et al. Doseintensive first-line chemotherapy with epirubicin and continuous infusion ifosfamide in adult patients with advanced soft tissue sarcomas: a phase II study. Eur J Cancer 1999;35:66-72.

3. Young RJ, Natukunda A, Litiere S, et al. First-line anthracycline-based chemotherapy for angiosarcoma and other soft tissue sarcoma subtypes: pooled analysis of eleven European Organisation for Research and Treatment of Cancer Soft Tissue and Bone Sarcoma Group trials. Eur J Cancer 2014;50:3178-86.

4. Savina M, Le Cesne A, Blay JY, et al. Patterns of care and outcomes of patients with METAstatic soft tissue SARComa in a real-life setting: the METASARC observational study. BMC Med 2017;15:78.

5. Seddon B, Strauss SJ, Whelan J, et al. Gemcitabine and docetaxel versus doxorubicin as first-line treatment in 
previously untreated advanced unresectable or metastatic soft-tissue sarcomas (GeDDiS): a randomised controlled phase 3 trial. Lancet Oncol 2017;18:1397-410.

6. Kawai A, Araki N, Sugiura H, et al. Trabectedin monotherapy after standard chemotherapy versus best supportive care in patients with advanced, translocationrelated sarcoma: a randomised, open-label, phase 2 study. Lancet Oncol 2015;16:406-16.

7. Schöffski P, Chawla S, Maki RG, et al. Eribulin versus dacarbazine in previously treated patients with advanced liposarcoma or leiomyosarcoma: a randomised, open-label, multicentre, phase 3 trial. Lancet 2016;387:1629-37.

8. Berry V, Basson L, Bogart E, et al. REGOSARC: Regorafenib versus placebo in doxorubicin-refractory soft-tissue sarcoma-A quality-adjusted time without symptoms of progression or toxicity analysis Cancer 2017;123:2294-302.

9. Stacchiotti S, Negri T, Zaffaroni N, et al. Sunitinib in advanced alveolar soft part sarcoma: evidence of a direct antitumor effect. Ann Oncol 2011;22:1682-90.

10. Van Der Graaf WT, Blay JY, Chawla SP, et al. Pazopanib for metastatic soft-tissue sarcoma (PALETTE): a randomised, double-blind, placebo-controlled phase 3 trial. Lancet 2012;379:1879-86.

11. Chi Y, Fang Z, Hong X, et al. Safety and efficacy of anlotinib, a multikinase angiogenesis inhibitor, in patients with refractory metastatic soft-tissue sarcoma. Clin Cancer Res 2018;24:5233-8.

12. Schachter J, Ribas A, Long GV, et al. Pembrolizumab versus ipilimumab for advanced melanoma: final overall survival results of a multicentre, randomised, open-label phase 3 study (KEYNOTE-006). Lancet 2017;390:1853-62.

13. Reck M, Rodríguez-Abreu D, Robinson AG, et al. Pembrolizumab versus chemotherapy for PD-L1positive non-small-cell lung cancer. $\mathrm{N}$ Engl J Med 2016;375:1823-33.

14. Plimack ER, Bellmunt J, Gupta S, et al. Safety and activity of pembrolizumab in patients with locally advanced or metastatic urothelial cancer (KEYNOTE-012): a nonrandomised, open-label, phase $1 \mathrm{~b}$ study. Lancet Oncol 2017;18:212-20.

15. Richard C, Fumet J-D, Chevrier S, et al. Exome analysis reveals genomic markers associated with better efficacy of nivolumab in lung cancer patients. Clin Cancer Res 2019;25:957-66.

16. Oda K, Kato K, Nakamura M, et al. Surface marker profiles on lung lymphocytes may predict the mechanism of immune-mediated pneumonitis triggered by tumorinfiltrating lymphocytes in lung cancer patients treated with pembrolizumab. Lung Cancer 2018;118:171-2.

17. Toulmonde M, Penel N, Adam J, et al. Use of PD-1 targeting, macrophage infiltration, and IDO pathway activation in sarcomas: a phase 2 clinical trial. JAMA Oncol 2018;4:93-7.

18. Paz-Ares L, Luft A, Vicente D, et al. Pembrolizumab plus chemotherapy for squamous non-small-cell lung cancer. $\mathrm{N}$ Engl J Med 2018;379:2040-51.

19. Rini BI, Plimack ER, Stus V, et al. Pembrolizumab plus axitinib versus sunitinib for advanced renal-cell carcinoma. N Engl J Med 2019;380:1116-27.

20. Shaverdian N, Lisberg AE, Bornazyan K, et al. Previous radiotherapy and the clinical activity and toxicity of pembrolizumab in the treatment of non-small-cell lung cancer: a secondary analysis of the KEYNOTE-001 phase 1 trial. Lancet Oncol 2017;18:895-903.

21. Kelly CM, Antonescu CR, Bowler T, et al. Objective response rate among patients with locally advanced or metastatic sarcoma treated with talimogene laherparepvec in combination with pembrolizumab: a phase 2 clinical trial. JAMA Oncol 2020;6:402-8.

22. Tawbi HA, Burgess M, Bolejack V, et al. Pembrolizumab in advanced soft-tissue sarcoma and bone sarcoma (SARC028): a multicentre, two-cohort, single-arm, openlabel, phase 2 trial. Lancet Oncol 2017;18:1493-501.

23. Wilky BA, Trucco MM, Subhawong TK, et al. Axitinib plus pembrolizumab in patients with advanced sarcomas including alveolar soft-part sarcoma: a single-centre, single-arm, phase 2 trial. Lancet Oncol 2019;20:837-48.

24. Groisberg R, Hong DS, Behrang A, et al. Characteristics and outcomes of patients with advanced sarcoma enrolled in early phase immunotherapy trials. J Immunother Cancer 2017;5:100.

25. Coindre JM, editor. New WHO classification of tumours of soft tissue and bone. Annales de pathologie; 2012.

26. Dueck AC, Mendoza TR, Mitchell SA, et al. Validity and reliability of the US National Cancer Institute's patientreported outcomes version of the common terminology criteria for adverse events (PRO-CTCAE). JAMA Oncol 2015;1:1051-9.

27. Ksienski D, Wai ES, Croteau N, et al. Pembrolizumab for advanced nonsmall cell lung cancer: Efficacy and safety in everyday clinical practice. Lung Cancer 2019;133:110-6.

28. Lin SY, Yang CY, Liao BC, et al. Tumor PD-L1 expression and clinical outcomes in advanced-stage nonsmall cell lung cancer patients treated with nivolumab 


\section{Page 12 of 12}

or pembrolizumab: real-world data in Taiwan. J Cancer 2018;9:1813-20.

29. Herbst RS, Baas P, Kim DW, et al. Pembrolizumab versus docetaxel for previously treated, PD-L1-positive, advanced non-small-cell lung cancer (KEYNOTE-010): a randomised controlled trial. Lancet 2016;387:1540-50.

30. Veenstra R, Kostine M, Cleton-Jansen AM, et al. Immune checkpoint inhibitors in sarcomas: in quest of predictive biomarkers. Lab Invest 2018;98:41-50.

31. D'Angelo SP, Shoushtari AN, Agaram NP, et al. Prevalence of tumor-infiltrating lymphocytes and PD-L1 expression in the soft tissue sarcoma microenvironment. Hum Pathol 2015;46:357-65.

32. Italiano A, Bellera C, D'Angelo S. PD1/PD-L1 targeting in advanced soft-tissue sarcomas: a pooled analysis of phase II trials. J Hematol Oncol 2020;13:55.

33. Wang PF, Chen Y, Song SY, et al. Immune-related adverse events associated with anti-PD-1/PD-L1 treatment for malignancies: a meta-analysis. Front Pharmacol 2017;8:730.

34. Kumar V, Chaudhary N, Garg M, et al. Current diagnosis

Cite this article as: Liu J, Fan Z, Bai C, Li S, Xue R, Gao T, Zhang L, Tan Z, Fang Z. Real-world experience with pembrolizumab in patients with advanced soft tissue sarcoma. Ann Transl Med 2021;9(4):339. doi: 10.21037/atm-21-49

\section{Liu et al. Pembrolizumab for advanced soft tissue sarcoma}

and management of immune related adverse events (irAEs) induced by immune checkpoint inhibitor therapy. Front Pharmacol 2017;8:49.

35. Gandhi L, Rodríguez-Abreu D, Gadgeel S, et al. Pembrolizumab plus chemotherapy in metastatic nonsmall-cell lung cancer. N Engl J Med 2018;378:2078-92.

36. Chowdhury S, McDermott DF, Voss MH, et al. A phase I/ II study to assess the safety and efficacy of pazopanib (PAZ) and pembrolizumab (PEM) in patients (pts) with advanced renal cell carcinoma (aRCC). American Society of Clinical Oncology; 2017.

37. Atkins MB, Plimack ER, Puzanov I, et al. Axitinib in combination with pembrolizumab in patients with advanced renal cell cancer: a non-randomised, open-label, dose-finding, and dose-expansion phase $1 \mathrm{~b}$ trial. Lancet Oncol 2018;19:405-15.

38. Makker V, Taylor MH, Aghajanian C, et al. Lenvatinib Plus Pembrolizumab in Patients With Advanced Endometrial Cancer. J Clin Oncol 2020;38:2981-92.

(English Language Editor: C. Betlazar-Maseh) 